\title{
A Fermi liquid model for the overdoped and optimally doped cuprate superconductors: scattering rate, susceptibility, spin resonance peak and superconducting transition
}

\author{
George Kastrinakis \\ Dept. of Physics, University of Illinois at Urbana-Champaign, Urbana, IL 61801, USA \\ and \\ Dept. of Chemical Engineering, University of Cambridge, Cambridge CB2 3RA, U.K. *
}

Physica C 340, 119 (2000)

(Received 14 Jan. 2000)

\begin{abstract}
We present a Fermi liquid model for the overdoped and optimally doped cuprate superconductors. For the normal state, we provide an analytic demonstration, backed by self-consistent Baym-Kadanoff (BK) numerical calculations, of the linear in temperature resistivity and linear in 1/energy optical conductivity, provided the interacting Fermi liquid has strong peaks in its density of states (van-Hove singularities in 2 dimensions) near the chemical potential $\mu$. Recent ARPES expts. by Valla et al., Science 285, 2110 (1999), and e-print cond-mat/0003407, directly support the linearity of the one-particle scattering rate everywhere in the Brillouin zone hereto obtained. We show that the origin of this linearity is the linear in energy term of the imaginary part of the carrier susceptibility. Moreover, we verify that the interactions tend to pin the van-Hove singularities close to $\mu$. We show that the low energy dependence of the susceptibility can have a purely fermionic origin. We introduce an ansatz for the susceptibility of the carriers, which we postulate to be enhanced in an additive manner due to the weak antiferromagnetic order of the $\mathrm{CuO}_{2}$ planes. Inter alia, this ansatz may explain the appearance of the spin resonance peak (observed in neutron scattering) in the normal state of the cuprates. Further, we obtain particularly high transition temperatures $T_{c}$ from our BK-Eliashberg scheme by using this ansatz: we have a $d_{x^{2}-y^{2}}$ gap with $T_{c}>120^{\circ} \mathrm{K}$ for nearest neighbour hopping $t=250 \mathrm{meV}$.
\end{abstract}

PACS numbers: 74.72.-h, 74.20.-z

\section{Introduction}

The nature of the many-body state of the cuprate superconductors is a central question for the understanding of these materials 1 ${ }_{0}$ E.g. one long standing puzzle has been the elucidation of the origin of the linear in temperature in-plane resistivity 1 and linear in 1/energy optical conductivity observed in the optimal doping regime and to a good extent in the overdoped regime. Vice-versa, the answer to this question should shed light on the character of the carriers and, subsequently, on the superconducting transition. Here we address these issues based on a minimum unconventional Fermi liquid modelE. Our model comprises strong peaks in its density of states (van-Hove singularities in 2 dimensions) near the chemical potential. We show that it accounts in a natural, comprehensive and internally consistent manner for several normal state characteristics. The introduction of an ansatz for the susceptibility of the carriers further allows us both to propose an explanation for the origin of the spin resonance peak and to obtain particularly high $d$-wave transition temperatures $T_{c}$. Overall, our results make a strong case for a Fermi liquid approach to the optimally doped and overdoped cuprates.

We perform a combination of analytical and numerical many-body calculations in the context of our model. The rest of this paper is organized as follows. In Section II we write our many-body approximation, which we used in our numerical calculations. We emphasize both that our treatment is relevant for overdoped and optimally doped cuprates (see Section VI for the underdoped regime) and that the results presented in Sections III-V depend only quantitavely and not qualitatively on the specific Hamiltonian and approximation thereof etc. In Section III we discuss our analytical and numerical results for the linear in $\max (T, \epsilon)$ scattering rate of the carriers, in connection with the existence of van Hove singularities (vHs) close to the chemical potential. We also show that a conductivity linear in $(1 / T, 1 / \epsilon)$ follows. In Section IV we discuss both the fermionic origin of the energy dependence of the Millis-MonienPines susceptibility and our ansatz of eq. (26) for the susceptibility of the carriers. We show that this ansatz may explain the appearance of the so called spin resonance peak, seen in neutron scattering experiments, in the normal state of the cuprates. In Section $\mathrm{V}$ we discuss the superconducting transition in the frame of the ansatz. Finally 
Section VI contains a summary of our results. In the Appendix we examine the role of doping-induced disorder on the carrier susceptibility.

\section{General framework}

We assume that we deal with a Fermi liquid, albeit an unconventional one, as will become apparent from our discussion of the scattering rate of the carriers below. We choose the 2-dimensional Hubbard Hamiltonian as a specific model for our numerical calculations (c.f. the last paragraph of Section I on this).

$$
H=\sum_{k, \sigma} \epsilon_{k} c_{k, \sigma}^{\dagger} c_{k, \sigma}+\frac{U}{2 N^{2}} \sum_{k, k^{\prime}, q, \sigma} c_{k+q, \sigma}^{\dagger} c_{k^{\prime}-q,-\sigma}^{\dagger} c_{k^{\prime},-\sigma} c_{k, \sigma}
$$

$c_{k, \sigma}^{\dagger}$ is an electron creation operator and $\epsilon_{k}$ is the electronic tight-binding dispersion suggested by angle-resolved photoemission (ARPES) experiments - e.g. seel - and LDA calculations

$$
\epsilon_{k}=-2 t\left(\cos k_{x}+\cos k_{y}\right)-4 t^{\prime} \cos k_{x} \cos k_{y}-2 t^{\prime \prime}\left(\cos 2 k_{x}+\cos 2 k_{y}\right) .
$$

It is assumed here that the lattice constant is equal to unity and is the same along the two crystal axis $a, b$ in the planes - hence $k_{x}, k_{y} \in[-\pi, \pi] . N \times N$ is the discretization of the Brillouin zone.

We consider the fluctuation-exchange diagrammatic approximation (FLEX) of Bickers, Scalapino and White $\mathrm{l}$ for the Hamiltonian, which consists in summing bubble and ladder diagrams. FLEX is a Baym-Kadanoff conserving approximations, meaning that there is a free energy functional $\Phi[G]$ of the Green' function $G$, such that the selfenergy $\Sigma$ is given by the relation $\Sigma=\delta \Phi[G] / \delta G$. We thus obtain a set of self-consistent equations for $G\left(k, \epsilon_{n}\right)$ and $\Sigma\left(k, \epsilon_{n}\right)$ :

$$
\begin{gathered}
G\left(k, \epsilon_{n}\right)^{-1}=G_{o}\left(k, \epsilon_{n}\right)^{-1}-\Sigma\left(k, \epsilon_{n}\right), \\
G_{o}\left(k, \epsilon_{n}\right)=\frac{1}{i \epsilon_{n}+\mu-\epsilon_{k}}, \\
\Sigma\left(k, \epsilon_{n}\right)=-\frac{T}{N^{2}} \sum_{q, \omega_{m}} V\left(q, \omega_{m}\right) G\left(k-q, \epsilon_{n}-\omega_{m}\right) .
\end{gathered}
$$

The potential $V\left(q, \omega_{m}\right)$ is given by

$$
\begin{gathered}
V\left(q, \omega_{m}\right)=V_{e x}\left(q, \omega_{m}\right)-V_{H}\left(q, \omega_{m}\right), \\
V_{e x}\left(q, \omega_{m}\right)=\frac{-U^{2} \chi_{o}\left(q, \omega_{m}\right)}{1-U^{2} \chi_{o}^{2}\left(q, \omega_{m}\right)}, \\
V_{H}\left(q, \omega_{m}\right)=\frac{U^{3} \chi_{o}^{2}\left(q, \omega_{m}\right)}{1-U \chi_{o}\left(q, \omega_{m}\right)},
\end{gathered}
$$

The susceptibility $\chi_{o}\left(q, \omega_{m}\right)$ is given by

$$
\chi_{o}\left(q, \omega_{m}\right)=-\left(T / N^{2}\right) \sum_{\epsilon_{n}, k} G\left(k+q, \epsilon_{n}+\omega_{m}\right) G\left(k, \epsilon_{n}\right) .
$$

$\mu$ is the chemical potential and the Matsubara frequencies are $\epsilon_{n}=(2 n+1) \pi T$ and $\omega_{m}=2 m \pi T$ for fermions and bosons, respectively. We solve numerically this self-consistent set of equations, working with a given number $M$ of Matsubara frequencies and discretization of the Brillouin zone $(M=256-480$ and $N \geq 64)$. 
There have been number of similar numerical calculations on the normal-phase and the superconducting transition of the cuprates 1 14, with FLEX being a particularly popular approach.

All the convolution operations are done by using the Fast Fourier Transform (FFT), in order to cut down calculation time. We use Padé approximants 15 to analytically continue our results to the real frequency axis.

\section{On the scattering rate of the cuprates}

We have analytically obtained a scattering rate linear in the maximum of the temperature $T$ or energy $\epsilon$, for a Fermi liquid with strong density of states peaks - van-Hove singularities (vHs) in 2-d - located at an energy $\epsilon_{v H}$ close to the chemical potential $\mu$.

The derivation relies on the relation (5) for the self-energy, which is valid quite generally in the frame of a BK approximation, irrespectively of the specific Hamiltonian and approximation thereof. See also the discussion following eq. (20). It can easily be shown 16 that $\operatorname{Im} \Sigma(k, \epsilon)$ is given by the following formula at finite temperature :

$$
\operatorname{Im} \Sigma^{R}(k, \epsilon)=\sum_{q, \omega} \operatorname{Im} G^{R}(q, \epsilon-\omega) \operatorname{Im} V^{R}(k-q, \omega)\{\operatorname{coth}(\omega / 2 T)+\tanh ((\epsilon-\omega) / 2 T)\}
$$

Taking

$$
\operatorname{Im} G^{R}(k, \epsilon)=-\pi \delta\left(s_{k, \epsilon}\right), \quad s_{k, \epsilon}=\epsilon+\mu-\epsilon_{k}-\operatorname{Re} \Sigma(k, \epsilon)
$$

we obtain

$$
\operatorname{Im} \Sigma^{R}(k, \epsilon)=-\pi \sum_{q} \operatorname{Im} V^{R}\left(k-q, s_{q, \epsilon}\right)\left\{\operatorname{coth}\left(s_{q, \epsilon} / 2 T\right)+\tanh \left(\left(\epsilon-s_{q, \epsilon}\right) / 2 T\right)\right\} .
$$

Setting $\operatorname{Im} G^{R}(k, \epsilon)$ equal to a delta function is a reasonable approximation for this purpose, in view of the typical sharp spike feature of $\operatorname{Im}^{R}(k, \epsilon)$ shown in fig. 1 - also see figs. 2 and 3, all of which are representative of our numerical solution of eqs. (3)-(9). Further, numerically $\operatorname{Im} G^{R}(k, \epsilon)$ is very small compared to the band energy for small couplings, and the difference of $\operatorname{Im} \Sigma(k, \epsilon)$, as seen in our numerical calculation, for small and large coupling constants is mostly quantitative rather than qualitative.

We write

$$
\operatorname{Im} V^{R}(q, x)=\sum_{n=0}^{\infty} \frac{V_{q}^{(2 n+1)}(0) x^{2 n+1}}{(2 n+1) !}
$$

where $V_{q}^{(n)}(0)$ is the $n$-th derivative of $\operatorname{Im}^{R}(q, \omega=0)$ with respect to $\omega$. This is true for an electronically mediated interaction, with a polarization which is a regular function of $\omega$ (see also eq. (20) below). There are only odd powers of $\omega$ in the series because the imaginary part of the susceptibility is an odd function of energy - e.g. ff. eq. (2.63) of Pines and Nozières17. One possible exception to this is given by Gonzalez, Guinea and Vozmediand 18 . The authors showed that for the underdoped LSCO-type Fermi surface (FS) and for momenta $K$ connecting two inflection points of the FS, the imaginary part of the susceptibility goes like $|\omega|^{1 / 4}$ in 2 dimensions. However, this fact will influence the final result for the scattering rate only for a small range of momenta $k$ satisfying $k=K+q_{o}$ - c.f. eqs. (14) and (15), and $q_{o}$ is given in the paragraph below. Moreover, we have already emphasized that our picture is not valid in the underdoped regime.

First we consider the low $T$ limit. The sum over $q$ is dominated by the van-Hove singularities at the points $q_{o}$. Assuming that $\epsilon_{v H}=\epsilon_{q_{o}}+\left\langle\operatorname{Re} \Sigma\left(q_{o}, \epsilon\right)\right\rangle$ [this relation is misprinted in the journal version of the paper] is close to $\mu$, the tanh has a vanishing contribution at the vicinity of $\epsilon_{q}+\operatorname{Re} \Sigma(q, \epsilon) \sim \mu$ (note that for $\epsilon_{q}+\operatorname{Re} \Sigma(q, \epsilon)<\mu$ and $\epsilon_{q}+\operatorname{Re} \Sigma(q, \epsilon)>\mu+\epsilon$ the contributions of tanh and coth annihilate each other in the low $T$ limit). Hence

$$
\operatorname{Im} \Sigma^{R}(k, \epsilon) \simeq-\pi \sum_{q \sim q_{o}} \sum_{n=0}^{\infty} \frac{V_{k-q}^{(2 n+1)}(0)\left(s_{q, \epsilon}\right)^{2 n+1}}{(2 n+1) !}
$$

For sufficiently small $V_{q}^{(n)}(0), \forall n>1$, we obtain 


$$
a_{k}=\pi \sum_{q \sim q_{o}} V_{k-q}^{(1)}(0) \gg \pi \sum_{q \sim q_{o}} \sum_{n=1}^{\infty} \frac{V_{k-q}^{(2 n+1)}(0)(\epsilon+c)^{2 n}}{(2 n+1) !},
$$

where $c=\mu-\epsilon_{v H}$. This relation is valid for $\epsilon+c<\epsilon_{c}$, where the latter is the characteristic energy beyond which the infinite sum on the right becomes comparable to the $V_{q}^{(1)}$ term. Also, for energies beyond the bandwidth $W(W=8 t$ for the non-interacting system), $\operatorname{Im} \chi_{o}$, and hence $\operatorname{Im} V$ (see below), decay to zero. These considerations yield the two energy crossovers

$$
\epsilon_{1}=\left|\mu-\epsilon_{v H}\right|, \quad \epsilon_{2}=\min \left\{\epsilon_{c}+\epsilon_{v H}-\mu, W+\epsilon_{v H}-\mu\right\}
$$

while the assumption above for $a_{k}$ leads to

$$
\operatorname{Im} \Sigma^{R}(k, \epsilon) \simeq-a_{k}(\epsilon+c), \epsilon_{1}<\epsilon<\epsilon_{2} .
$$

For $\epsilon>\epsilon_{2} \operatorname{Im} \Sigma$ gradually decreases, due to the finite bandwidth of the system. Finally, we note that if the Fermi surface approaches a van-Hove singularity at $q_{o}, V_{k-q}^{(1)}(0)$ should become bigger, being proportional to $1 /\left(\vec{\nabla} \epsilon_{k_{F}} \vec{k}_{F}\right)$ (as implied by the standard Fermi liquid result for the imaginary part of the susceptibility 17 - see the discussion below on the susceptibility of the cuprates).

We consider now the high temperature limit $T>\left(\mu-\epsilon_{v H}\right) / 419$. We see immediately that

$$
\operatorname{Im} \Sigma^{R}(k, \epsilon)=-\pi \sum_{q} \operatorname{Im} V^{R}\left(k-q, s_{q, \epsilon}\right)\left\{2 T / s_{q, \epsilon}+O\left(s_{q, \epsilon} / 2 T\right)\right\} .
$$

(Note that the term of order $T$ of this sum is reminiscent of the left-hand side of the sum rule - c.f. Pines and Nozières 17 - $\lim _{q \rightarrow 0} \int_{0}^{\infty} d \omega \operatorname{Im} \chi_{o}(q, \omega)|\varepsilon(q, \omega)|^{2} / \omega=-N \pi / m c_{s}^{2}$, with $N$ being the total particle number, $c_{s}$ the speed of sound, $m$ the effective mass, and $\varepsilon(q, \omega)$ the dielectric function.) The sum is dominated by the van-Hove singularities at the points $q_{o}$, thus yielding

$$
\operatorname{Im} \Sigma^{R}(k, \epsilon) \simeq-2 T \pi \sum_{q \sim q_{o}} V_{k-q}^{(1)}(0)=-2 a_{k} T
$$

Here we made use of the condition above for $a_{k}$. In addition, it is straightforward to see from our analytic treatment that $\operatorname{Im} \Sigma^{R} \propto x^{2}, x=\max \{T, \epsilon\}$, when both $T, \epsilon \rightarrow 0$. In all respects we have a genuine Fermi liquid.

Note added: ARPES expts. by Valla et al., Science 285, 2110 (1999), and preprint cond-mat/0003407, have very recently shown that in optimally doped $\mathrm{Bi}_{2} \mathrm{Sr}_{2} \mathrm{CaCu}_{2} \mathrm{O}_{8+y}$ the one-particle scattering rate is linear in $\max \{T, \epsilon\}$ over most of the Fermi surface, in support of our picture.

A brief comment here. It has been known long ago - see e.g.20 - that the scattering rate becomes linear in $T$ for $T>\omega_{B} / 4$, with $\omega_{B}$ being the characteristic boson frequency mediating the carrier interaction. Our treatment shows that $\omega_{B}$ here is nothing else but the fermionic energy $\mu-\epsilon_{v H}$.

The prefactors in the r.h.s. of eqs. (17) and (19) differ by a factor of 2. This is in agreement with experiments in $\mathrm{YBa}_{2} \mathrm{Cu}_{3} \mathrm{O}_{7-\delta}$ and $\mathrm{Bi}_{2} \mathrm{Sr}_{2} \mathrm{CaCu}_{2} \mathrm{O}_{8+y}$, 2 , where the factor is found to be in the range 2.1 - 2.2 . We note that the "marginal Fermi liquid" phenomenology of Varma, Littlewood, Schmitt-Rink, Abrahams and Ruckenstein 22 gives a factor of $\pi$ instead.

We emphasize that the $T$ and $\epsilon$ dependence of the result are independent of $k$ - thus leading necessarily to a linear in $T$ resistivity and a linear in $1 / \epsilon$ optical conductivity, even with inclusion of vertex corrections in the calculation. The reason for this being that $\{T, \epsilon\}$ are obtained as overall prefactors, for the relevant $T$ and $\epsilon$ regimes, in such calculations. E.g. the Kubo formula yields $\sigma(\omega)=\left(e^{2} / \omega\right) \sum_{k, \epsilon} v_{k}^{2} G(k, \epsilon+\omega) G(k, \epsilon)[1+S(k, \epsilon)][f(\epsilon+\omega)-f(\epsilon)]$, where $v_{k}$ is the group velocity, $S$ includes vertex corrections from the Ward identity, and $f$ is the Fermi occupation factor. The main $T$ and $\omega$ dependence in the integrand is in the one-particle self-energy in $G$ and in $f$. Doing the $k$ sum, we get the dominant contribution from the poles of $G$. Now, the one-particle scattering rate is linear in $\max (T, \epsilon)$ everywhere in the Brillouin zone, and this linear dependence appears in the denominator of $\sigma$. Here we assumed that the vertex corrections do not have a strong temperature dependence over a substantial part of the Brillouin zone. Indeed, Kontani, Kanki and Ueda 33 have recently shown numerically, in the frame of the FLEX approximation, that vertex corrections are small for the resistivity, and do not change its $T$ dependence.

Hlubina and Rice 24 considered analytically a model of interacting fermions with a vHs close to $\mu$. However, they find a scattering rate similar to ours only close to the $\mathrm{vH}$ region, and different otherwise. As a result, their resistivity goes like $T^{2} \ln ^{2}(1 / T)$. In their 'hot' and 'cold' spots scenario, relying on strong scattering off antiferromagnetic fluctuations, they obtain an average scattering rate similar to ours, and numerically a linear in $T$ resistivity (however, 
they seem to assume that the group velocity is finite along the whole Fermi surface - cf. betweeneqs. (2.5) and (2.6) ol24). Similar results are also obtained in the antiferromagnetic scenario of Pines and Stojkovic25.

A note on phonons. As they form a - presumably small- part of the effective potential $V$, they provide necessary momentum dissipation, yielding a finite resistivity. However, the linear $T$ dependence of the latter is not specifically influenced by phonons in our model.

Returning to the derivation of the scattering rate above, we observe that the overall behavior of $\operatorname{Im} V$ closely follows $\operatorname{Im} \chi_{o}(q, \omega)$, as

$$
\operatorname{Im} V(q, \omega)=\operatorname{Im} \chi_{o}(q, \omega)|\varepsilon(q, \omega)|^{2},
$$

$\operatorname{Im} \chi_{o}$ is odd in $\omega$, while $|\varepsilon|^{2}$ is even. Eq. (20) follows from any screened interaction between the carriers. Hence the argument for the linear in energy and temperature behavior of $\tau^{-1}(T, \epsilon)$ is equally generic. It relies essentially on a large coefficient for the linear in energy term of $\operatorname{ImV}$ - i.e. of $\operatorname{Im} \chi_{o}$ - and the presence of van-Hove singularities near the Fermi surface. The result holds regardless of the dimensionality of the system. However, it is important that a significant part of the spectral weight be included in the strong peaks of the density of states lying close to $\mu$.

What is more, in our self-consistent numerical solution we observe that the energy $\epsilon_{v H}$ of the singularities is pushed by the interactions close to the chemical potential - see fig. 4 . This result is especially pronounced when we use the ansatz for the susceptibility of the carriers of eq. (26) below. Then we find typically for $n \sim 0.87-0.95$ and for a broad range of $t^{\prime}, t^{\prime \prime}, U$

$$
\mu-\epsilon_{v H} \leq t / 20
$$

The shape of the self-energy $\Sigma(k, \epsilon)$ of the interacting system is responsible for the modification of the density of states $N(\epsilon)$, through the relation $N(\epsilon)=-\operatorname{Tr} \operatorname{Im} G(k, \epsilon) / \pi$. A trend for the transfer of the spectral weight is indicated by the fact that $\operatorname{Im} \Sigma(k, \epsilon)$ has a peak below $\mu$ and a dip above it. The numerical result concerning the approachment between $\epsilon_{v H}$ and $\mu$ has been known for some years. Si and Levin 20 and Newns, Pattnaik and Tsuei26 observed the pinning of the vHs close to $\mu$ by using a $U \rightarrow \infty$ mean field slave boson approximation of a model with $\mathrm{Cu} 3 \mathrm{~d}$ and $\mathrm{O} 2 \mathrm{p}$ orbitals. Recently, Gonzalez, Guinea and Vozmediano27 were able to obtain analytically the essential part of the approachment between the vHs and $\mu$ with a first order renormalization group treatment in the context of the Hubbard model. A review of related work in the frame of the so-called van-Hove scenario has been given by Markiewic 22. This pinning of the vHs close to $\mu$ seems to be a plausible explanation for the common characteristic of a good many cuprates whose van-Hove singularities are located between 10-30 meV below the Fermi surface 29 (see also the next section).

It is interesting that the electron doped $\mathrm{Nd}_{2-x} \mathrm{Ce}_{x} \mathrm{CuO}_{4+\delta}$ which has a van-Hove singularity much below the Fermi surface, i.e. at approximately $\mu-350 \mathrm{meV}$, as shown by ARPES 30 , has a usual Fermi liquid $\tau^{-1}(T)=$ const. $T^{2} \ln (T) 31$. This lends support to the picture described above. Along the same line, the resistivity of $\mathrm{Tl}_{2} \mathrm{Ba}_{2} \mathrm{CuO}_{6}+\mathrm{Tl}_{\mathrm{g}}(\mathrm{T} 201)$ switches over from linear to quadratic with increasing doping from the optimal to the overdoped regime 32 , which we suspect to be an indication of the vHs moving well away from $\mu$.

Finally, in further support of the relevance of the vHs in the transport properties of the cuprates, Newns et. al 33 and McIntosh and Kaiser 34 have shown that the thermal conductivity of the cuprates can be well accounted for if the vHs are located very close to the Fermi surface, as discussed above.

The numerical solution of the many-body system always corroborates our analytical result for the self energy at finite temperature. $\operatorname{Im} \Sigma(k, \epsilon)$ turns out to be essentially linear in energy in the interval $\epsilon_{1}<\epsilon<\epsilon_{2}$. A linear dependence of $\operatorname{In} \Sigma(k, \epsilon)$ as a function of either $T$ or $\epsilon$ was also obtained in the numerical work of Beere and Annett 35 , Kontani et al.23 and $\mathrm{Si}$ and LevinE2. Note that in figs. 2 and 3 we show the self-energy for the set of the system parameters which yields the highest transition temperature $T_{c}$, if use of the ansatz of eq. (26) is made. The linearity of $\operatorname{Im} \Sigma(k, \epsilon)$ with $\epsilon$ is even more pronounced for other combinations of $t^{\prime}, t^{\prime \prime}$ and $n$. $\operatorname{Im} \Sigma(k, \epsilon)$ has always the correct parabolic Fermi liquid bevahior for $\epsilon \rightarrow 0$. Furthermore, the energy interval of linear behavior expands as the energy $\epsilon_{v H}$ of the (extended) van-Hove singularities at the (vicinity of the) points $q_{o}=( \pm \pi, 0),(0, \pm \pi)$ approaches $\mu$.

Another feature of the density of states as seen in our treatment - c.f. fig. 4 - is the following. The non-interacting density of states has two minor peaks at the bottom and top of the spectrum respectively (in fig. 4 the top one is a vHs). As the strength of the interaction increases, these two peaks are washed out, as a result of the self-energy which becomes substantial in magnitude for energies away from $\mu$ - c.f. figs. 2 and 3.

At the moment it is not clear whether the present mechanism of the linear scattering rate can explain the experimentally observed $T^{3}$ dependence of the Hall resistivity of the cuprates. A way to explain it has been found by Stojkovic and Pines 25, using an electron interaction peaked at $Q=( \pm \pi, \pm \pi)$. Their argument can be slightly modified, so that it works for our form of the electron potential $V$ - given by eq. (6) - but with a modified effective $\chi_{o}$ peaked at Q, as we propose in the next section - c.f. eq. (26) and below. Kontani et al.23 have shown that vertex corrections in the 
frame of FLEX have a drastic influence on the $T$ dependence of the Hall resistivity, in marked contrast to the case of the longitudinal resistivity.

\section{On the susceptibility of the cuprates}

The low energy dependence of the susceptibility of the cuprates. The Millis-Monien-Pines susceptibility 36 , 3

$$
\chi_{M M P}(q, \omega)=\frac{X_{1} \xi^{2}}{1+\xi^{2}(q-Q)^{2}-i \omega / \omega_{S F}},
$$

has been used to fit the low energy part of the susceptibility of the cuprates in both NMR rate and inelastic neutron scattering (INS) experiments. Here $Q=( \pm \pi, \pm \pi)$. The short range antiferromagnetic (AF) order, a remnant of the parent antiferromagnetic materials, with correlation length $\xi$, is responsible for the peak of the susceptibility for $q$ near $Q$. Typically $\xi$ is of the order of the lattice constant ( $\xi$ decreases as the doping increases, and e.g. $\xi \simeq 2$ for optimally doped $\left.\mathrm{YBa}_{2} \mathrm{Cu}_{3} \mathrm{O}_{7-\delta}\right)$, while $\omega_{S F} \approx 10-40 \mathrm{meV}$.

The origin of the small magnitude of $\omega_{S F}$ has remained elusive thus far. E.g. Sachdev, Chubukov and Sokol have interpreted it as a damped spin wave mode 37 . Spin waves are clearly observable in underdoped cuprates. However to date there is no experimental proof that they are strong enough in the normal phase of the optimally doped and overdoped regimes. The proximity of the system to an antiferromagnetic instability, i.e. $\bar{V}_{Q} \chi(Q, \omega) \simeq 1$, where $\bar{V}_{q}$ and $\chi(q, \omega)$ are some appropriate coupling and susceptibility respectively, can in principle explain the small magnitude of $\omega_{S F}$, as pointed out by Millis, Monien and Pines 36 .

Here we propose that an alternative explanation - which may coexist with the latter - is the following fermionic origin for $\omega_{S F}$.

First however, let us present a proposal for the susceptibility which has been put forward by Onufrieva and RossatMignod 38. This starts by viewing the $\mathrm{CuO}_{2}$ planes as a lattice of plaquettes centered on the copper site with four nearest neighbour oxygen sites. A Hamiltonian $\mathcal{H}$, reminiscent of but more comprehensive than the one of the $t-J$ model, was introduced in terms of the Hubbard operators. In this formulation, the itinerant carriers which propagate via $\mathrm{Cu}$ spin flips are clearly separate objects from the localized $\mathrm{Cu}$ spins with short range AF order. A diagrammatic approach was developed in the frame of $\mathcal{H}$, leading to the following RPA-type total susceptibility

$$
\chi_{t}(q, \omega)=\frac{\chi_{A F}(q, \omega)+\chi_{F}(q, \omega)}{1+J_{q}\left(\chi_{A F}(q, \omega)+\chi_{F}(q, \omega)\right)} .
$$

$J_{q}$ is the effective $\mathrm{Cu}$ spin exchange interaction, $\chi_{F}$ is a purely fermionic susceptibility and $\chi_{A F}(q, \omega)$ is due to the localized spins. $\chi_{t}(q, \omega)$ encompasses in an appealing way the idea of the entangled carrier-spin dynamics in the cuprates. Furthermore, this approach is able to account to a good extent for the variation of the total susceptibility as a function of doping and temperature, as measured by INS.

Now, we use the result for $\chi_{t}$ above with

$$
\chi_{A F}(q, \omega)=\frac{\chi_{1} \xi^{2}}{1+\xi^{2}(q-Q)^{2}-f(\omega)}, \quad \chi_{F}(q, \omega)=\chi_{F_{o}}\left(1+i \omega / \omega_{o}+O\left(\omega^{2}\right)\right), q \rightarrow Q .
$$

Let us suppose that $f(\omega)=i \omega / \omega_{S}$. If $\omega_{S} \gg \omega_{o}$ and $J_{Q} \xi^{2} \chi_{1}<1$, and taking $\chi_{F}(q, \omega) \equiv \chi_{o}(q, \omega)$ (as given by eq. (9)), we essentially recover $\chi_{M M P}(q, \omega)$ - which is itself an approximate form of the true susceptibility - with

$$
\omega_{S F} \rightarrow \bar{\omega}(q)=\frac{\omega_{o} \omega_{S}}{\omega_{o}+\omega_{S} J_{q} \chi_{o}^{2}\left(1+\xi^{2}(q-Q)^{2}\right) /\left(\xi^{2} \chi_{1}\right)}
$$

From the numerical solution of our system, we easily obtain values of $\omega_{o}$ comparable to the experimentally relevant ones, when the van-Hove energy $\epsilon_{v H}$ is near $\mu$, with $\omega_{o}$ scaling quickly towards zero as $\mu-\epsilon_{\mathrm{g} H} \rightarrow 0$. Hence $\omega_{o}$ can be interpreted as $\omega_{o}\left(\vec{q}_{F}\right)=\vec{\nabla} \epsilon_{q_{F}} \vec{q}_{F}$ - c.f. the non-interacting Fermi liquid result $\omega_{o}(q)=v_{F} q \mathbb{1}$. The small difference $\mu-\epsilon_{v H}$ is observed in a good number of cuprates. E.g. in ref.29 there is a compilation of several cuprates, the vanHove singularities of which are located between $10-30 \mathrm{meV}$ below the Fermi level (c.f. the discussion in the previous section). Also, Blumberg, Stojkovic and Klein (BSK) 6 suggested that this characteristic may be true irrespective of the doping, as long as the latter is appropriate for superconductivity. This is based on ARPES experiments on the bilayer $\mathrm{YBa}_{2} \mathrm{Cu}_{3} \mathrm{O}_{7-\delta}$. ARPES remains the best diagnostic probe for the Fermi surface of the cuprates. Yet it has not 
proved possible to perform measurements on many other compounds, especially the monolayers such as $\mathrm{Tl}_{2} \mathrm{Ba}_{2} \mathrm{CuO}_{6+\delta}$ etc. The point here is the following. By fitting the ARPES data BSK show that one of the two effective bands - the anti-bonding one - formed by hybridization of the two layers by interlayer coupling has a chemical potential only some $20-50 \mathrm{meV}$ above the van-Hove singularity at $(0, \pi)$, irrespective of the doping regime. It is then clear that these carriers, with a large density of states, give rise to a small $\omega_{o}$ as discussed above. Hence it is very interesting to know how universal this band-structure characteristic of the cuprates is, as it may explain naturally the magnitude of $\bar{\omega}$. Furthermore, it would be interesting to determine experimentally, e.g. by INS, the value of $\bar{\omega}$ for $\mathrm{Nd}_{2-x} \mathrm{Ce}_{x} \mathrm{CuO}_{4+\delta}$. In that case, $\omega_{o}$ should be enhanced as a result of the van-Hove singularities being far away from the Fermi surface.

In the Appendix we discuss the (non)influence of weak disorder on the value of $\omega_{0}$.

The antiferromagnetic ansatz for the carrier susceptibility. We thereby propose that the effective noninteracting susceptibility (i.e. without interaction lines connecting the particle-hole lines) of the carriers is given by the following ansatz

$$
\chi_{o}(q, \omega) \rightarrow \chi_{o}^{e f f}(q, \omega)=\chi_{o}(q, \omega)+a \chi_{A F}(q, \omega) .
$$

$\chi_{o}$ is given by eq. (9) above, $\chi_{A F}$ is the antiferromagnetic susceptibility of the localized $\mathrm{Cu}$ spins and $0<a<1$ is a dimensionless weighting factor, which in principle depends on doping, band structure, temperature (presumably a decreasing function of the latter) etc. The intuitive idea is that the carriers shouldperceive the ordered antiferromagnetic background of the $\mathrm{CuO}_{2}$ planes, even in the absence of phase separation 39 t 1 . In this way, the fermionic susceptibility acquires an antiferromagnetic enhancement, which may then influence the effective carrier potential and pairing, through an electronically mediated interaction - see also the next section. Furthermore, $\chi_{o}^{e f f}$ can explain the temperature dependence of the Hall resistivity of the cuprates, as we mentioned at the end of the previous section on the scattering rate. Finally, we note that with this effective $\chi_{o}^{\text {eff }}$ our many-body approximation remains conserving, since the relation $\Sigma=\delta F / \delta G$ between the self-energy, the free energy and the Green's function is still walid, as $\chi_{A F}$ does not depend on $G$. We note that this is consistent with the work of Onufrieva and Rossat-Mignod 38 mentioned above. The carriers and the localized spins form two distinct, albeit interrelated, systems. The additive form of the ansatz is also compatible in spirit with $\chi_{t}(q, \omega)$ of eq. (23).

We mention here the alternative treatment of the spin and charge susceptibilities of Imada, Fujimori and Tokura 42.

Taking the ansatz of eq. (26) at face value in the context of our many body scheme means that both the charge and spin susceptibility of the carriers acquire an AF enhancement. Currently we cannot prove this ansatz. We emphasize that our ansatz can be taken to apply only for the spin susceptibility of the carriers. In that case, in the frame of our Baym-Kadanoff scheme, only $\chi_{o}(q, \omega)$ entering the ladder diagrams with opposite particle-hole spins would be replaced by $\chi_{o}^{e f f}$. Quantitatively, the differerence between this case and the case in which both charge and spin susceptibilities are enhanced is small (for relevant values of the AF enhancement) - c.f. the discussion on the critical temperature $T_{c}$ in the next section.

In our numerical implementation, we consider two similar forms for the AF susceptibility, namely (A)

$$
\chi_{A F}^{A}\left(q, \omega_{m}\right)=X_{o} \sum_{i=1}^{4} \Gamma_{i}^{-1} \theta\left(\omega_{c}-\left|\omega_{m}\right|\right),
$$

with $\Gamma_{i}=\xi^{-2}+\left(q-Q_{i}\right)^{2}, \omega_{c}$ being a cut-off, and (B)

$$
\chi_{A F}^{B}\left(q, \omega_{m}\right)=X_{o} D \sum_{i=1}^{4} \frac{\omega_{m}-\left(2 \omega_{m} / \pi\right) \arctan \left(\omega_{m} / D\right)-\Gamma_{i} D+\left(2 \Gamma_{i} D / \pi\right) \arctan \left(\Gamma_{i}\right)}{\omega_{m}^{2}-\left(\Gamma_{i} D\right)^{2}},
$$

with $Q_{i}=( \pm \pi, \pm \pi)$ and $D$ being a cut-off frequency, above which $\operatorname{Im} \chi_{A F}(q, \omega)=0$. Form (B) has appeared in 43 . Here the characteristic spin wave frequency obeys $\omega_{S} \propto \xi^{-z}$, and the $z=2$ scaling regime has been assumed, in agreement with the analysis of Sokol and Pines for the optimally doped and overdoped regime of $\mathrm{ZBa}_{2} \mathrm{Cu}_{3} \mathrm{O}_{7-\delta}$ etc. 44

The spin resonance peak of the cuprates. INS experiments in $\mathrm{YBa}_{2} \mathrm{Cu}_{3} \mathrm{O}_{7-\delta}$ - see e.g. 45 and therein - and $\mathrm{Bi}_{2} \mathrm{Sr}_{2} \mathrm{CaCu}_{2} \mathrm{O}_{8+y} 46$ have revealed the existence of a strong peak in the spin triplet channel, centered at $Q=( \pm \pi, \pm \pi)$ and at a characteristic resonance energy $\omega_{R} \simeq 30-40 \mathrm{meV}$. Although this peak is usually seen exclysively in the superconducting state, it has been observed up to temperatures $\sim 250^{\circ} \mathrm{K}$ in $\mathrm{YBa}_{2}\left(\mathrm{Cu}_{0.995} \mathrm{Zn}_{0.005}\right)_{3} \mathrm{O}_{7} 45$ for $\omega_{R} \sim{ }^{4}$ $\mathrm{meV}$. Interestingly, this fact cannot be accounted for by most of the theoretical models so far available - see e.g. 45,46 for refs. - as these models require the onset of superconductivity. An exception is the model of Bulut 4 , which, however, differs drastically from ours.

The use of the ansatz of eq. (26) in the spin channel only may account in a natural way for the appearance of the resonance peak in the normal state through a bilayer effect. Both $\mathrm{YBa}_{2} \mathrm{Cu}_{3} \mathrm{O}_{7-\delta}$ and $\mathrm{Bi}_{2} \mathrm{Sr}_{2} \mathrm{CaCu}_{2} \mathrm{O}_{8+y}$ are 
bilayer materials. Here one can define bonding and antibonding bands14 $\epsilon_{ \pm k}=\epsilon_{k} \pm t_{\perp}(k), t_{\perp}(k)$ being the $k$-dependent interlayer hopping element. Further, one defines the susceptibilities $\chi_{o \pm}(q, \omega)=\operatorname{Tr}\left[G_{+}(+q,+\omega) G_{ \pm}+G_{-}(+q,+\omega) G_{\mp}\right]$. When $\epsilon_{v H}-\mu$ is small, $\chi_{o-}(q, \omega)$ has a narrow peak at $q=Q$ and $\omega \simeq \Delta$, where $\Delta$ is the bilayer splitting at $q \sim q_{o}=(\pi, 0),(0, \pi)$, i.e. the van Hove neighbourhood. Liechtenstein et al.t4 have shown in the frame of the FLEX approximation that $\Delta$ is drastically reduced for a finite interaction, compared to the non-interacting value $\Delta_{o}=2 t_{\perp}\left(q=q_{o}\right)$, such that it becomes comparable to the experimental $\omega_{R}$. Our ansatz can be taken to apply to $\chi_{o \pm}$, yielding $\chi_{o \pm} \rightarrow \chi_{o \pm}^{\text {eff }}=\chi_{o \pm}+a \chi_{A F}$. As a result $\chi_{-}(q, \omega)=\chi_{o-}^{\text {eff }}(q, \omega) /\left(1-U \chi_{o-}^{\text {eff }}(q, \omega)\right)$ is strongly peaked at $Q$ for an energy $\omega_{c R} \sim \Delta$, and may account for the experimental observations. Of course we require $\chi_{o-}>\chi_{o+}$ for this to work, which is valid for a range of the parameters $t^{\prime}, t^{\prime \prime}, n$.

One can ask the question: why does the resonance peak not appear in the normal state in general? As demonstrated here, the amplitude of the AF enhancement needs to be sufficiently large for the peak to be visible. Zn is known to enhance AF fluctuations in the $\mathrm{CuO}_{2}$ planes - e.g 4 , which if interpreted as yielding a larger $\left(a \chi_{A F}\right)$ contribution in $\chi_{o}^{e f f}$, is in agreement with the results above. On the other hand, as we discuss in the next section on the superconducting transition, too strong a factor $\left(a \chi_{A F}\right)$ reduces $T_{c}-$ c.f. $T_{c}=93^{\circ} \mathrm{K}$ for the pure $\mathrm{YBa}_{2} \mathrm{Cu}_{3} \mathrm{O}_{7}$ versus $T_{c}=87^{\circ} \mathrm{K}$ for the $\mathrm{Zn}$ doped material mentioned above (also see the last paragraph of section $\mathrm{V}$ ).

\section{On the superconducting transition of the cuprates}

With the solution of the normal system at hand, we solve the gap (Eliashberg) equation 19

$$
\Delta\left(k, \epsilon_{n}\right)=-\frac{T}{N^{2}} \sum_{k^{\prime}, \epsilon_{n}^{\prime}} V_{p}\left(k-k^{\prime}, \epsilon_{n}-\epsilon_{n}^{\prime}\right) G\left(k^{\prime}, \epsilon_{n}^{\prime}\right) G\left(-k^{\prime},-\epsilon_{n}^{\prime}\right) \Delta\left(k^{\prime}, \epsilon_{n}^{\prime}\right) .
$$

This form of the equation is valid close to the transition temperature $T_{c}$ only. The pairing potential $V_{p}$ is given by

$$
\begin{gathered}
V_{p}\left(q, \omega_{m}\right)=V_{x}\left(q, \omega_{m}\right)+V_{h}\left(q, \omega_{m}\right) \\
V_{x}\left(q, \omega_{m}\right)=\frac{U}{1-U^{2} \chi_{o}^{2}\left(q, \omega_{m}\right)} \\
V_{h}\left(q, \omega_{m}\right)=\frac{U^{2} \chi_{o}\left(q, \omega_{m}\right)}{1-U \chi_{o}\left(q, \omega_{m}\right)}
\end{gathered}
$$

We have assumed that the gap is an even function of both its momentum and energy arguments in order to write $V_{p}\left(q, \omega_{m}\right)$ in this form. We also assumed that the gap is spin singlet, as Knight shiftexperiments have shown 2 . The superconducting state of the cuprates is probably a generalized BCS state see e.g. 00 - with the transition being due to a momentum anisotropic potential, as envisaged by Kohn and Luttinger 51 .

The symmetry of the gap is determined by the exact shape and sign of $V_{p}$. Based on symmetry grounds as well as on experimental evidence, we expect a $d_{x^{2}-y^{2}}$ or a $s$ wave gap - but see also 2 . (In principle, higher order even angular momentum harmonics are also possible.) The highest $T_{c}$ 's correspond to a $d_{x^{2}-y^{2}}$ gap, and are obtained by including an antiferromagnetically enhanced susceptibility in the calculation, following our ansatz of eq. (26). In passing, let us note that in general the proximity of $\epsilon_{v H}$ to $\mu$ plays a less significant role than the ansatz in raising $T$. - but c.f. below. Nevertheless we also obtain a $s$-wave gap under the same conditions, but with a much lower $T_{c}^{\prime} 53$. This is consistent with the experimental situation. Most of the cuprates appear to have a $d_{x^{2}-y^{2}}$ gap at $T_{c}$ - e.g. c.f.t. Experiments point to the opening of a secondary order parameter gap at $T_{c}^{\prime} \ll T_{c} 5456$.

We note here that the van-Hove singularities at $q_{o}$ tend to suppress a $s$-wave gap if the pairing potential $V_{p}>0$ ( $V_{p}$ defined above is negative for sufficiently large and negative $U$ and/or an appropriate phonon coupling, and positive otherwise). The (likely) $s$-wave gap of $\mathrm{Nd}_{2-x} \mathrm{Ce}_{x} \mathrm{CuO}_{4+\delta}$ could originate from e.g. the fact that the van-Hove singularities are $350 \mathrm{meV}$ below the Fermi surface, and hence ineffective here, or from $V_{p}<0$ for some relevant parts of the phase space in this material, or possibly from both facts. Yet another possibility which may coexist with the above is that $V_{p}$ is strongly peaked close to zero momentum owing to the band structure of this material. All these factors can result in the $s$-wave $T_{c}$ being higher than the $d$-wave $T_{c}$, and hence in the dominance of the former channel over the latter. 
We obtain the following (near) optimum set of parameters for the $d$-wave $T_{c}$. We take $t=250 \mathrm{meV}$ and $\xi=1$ (increasing the latter leads to a reduction of $T_{c}$, see below). For form (A) - eq. (27) - we obtain $T_{c} \simeq 125^{\circ} K$, for $a X_{o}=0.2 e V^{-1}, \omega_{c}=4 t, t^{\prime}=-0.11 t, t^{\prime \prime}=0.5 t, U=1.27 e V$ and $n=0.88$. For form (B) - eq. (28) - we obtain $T_{c} \simeq 105^{\circ} \mathrm{K}$, for $a X_{o}=0.5 \mathrm{eV}^{-1}, D=32 t, t^{\prime}=-0.11 t, t^{\prime \prime}=0.25 t, U=1.47 \mathrm{eV}$ and $n=0.91$. The variation of $T_{c}$ in ${ }^{o} K$ with $\xi$ is as follows for this last set of parameters : $(94,1.5),(91,2)(90,3),(88,5)$.

Monthoux and Pines obtained similarly high transition temperatures 10 with their approach, which uses $\mathcal{V}(q, \omega)=$ $g^{2} \chi_{M M P}(q, \omega)$ as the effective carrier-carrier potential. Their approach has no room for the Hubbard $U$ and the subsequent screened carrier-carrier interaction though. Overall, our approach yields significantly enhanced $T_{c}$ 's compared to the standard FLEX-type treatments 5 .

We obtain an optimum value of $n$ for the following reason. $\chi_{o}$ - and hence $\chi_{o}^{e f f}$ - is a decreasing function of $n$. We allow the coupling to increase up until $b=U \chi_{o}^{e f f}(Q, \omega=0)$ saturates to a value close to and below unity. Then $V$ and $V_{p}$ (given by eqs. (6) and (32) respectively) are increasing with $n$. And so does the characteristic scattering rate entering $G$. Thus the optimum value of $n$ for the highest $T_{c}$ corresponds to the overall highest kernel $V_{p}|G|^{2}$ in the gap equation (29).

Further, we have done an extensive search of the parameter space to locate the optimum parameter set for the highest $T_{c}$. From our data it appears that the variation of $T_{c}$ as a function of the system parameters is smooth and that the optimum parameter set above is a global optimum58.

Assuming that only the spin susceptibility acquires an AF enhancement according to our ansatz, yields a $T_{c}$ which is lower by $9 \%$ for $\xi=1$, but only lower by $2 \%$ for $\xi=3$, if we make use of $\chi_{A F}^{B}$, with the optimum parameters above. The variation of $T_{c}$ with $\xi$ is as follows here : $(96,1),(92,1.5),(90,2),(89,3),(88,5)$.

There is an optimum value of $a$ for the maximum attainable $T_{c}$. This is again due to the form of the pairing potential $V_{p}$ above : the AF instability condition allows $U \chi_{o}^{e f f}<1$ only. Now, for a given value of the latter product, the highest $V_{p}$ - which in principle yields the highest $T_{c}$ as well (c.f. above) - will correspond to the highest possible $U$. This in turn corresponds to a smaller $\chi_{o}^{e f f}$, and hence to a small but finite optimum $a$. In the next paragraph we discuss relevant experimental evidence.

Zheng, Kitaoka, Ishida and Asayama59 made a very interesting empirical observation. Namely, among the hole doped cuprates, the highest $T_{c}$ 's correspond to a combination of both optimum total carrier concentration $n_{x^{2}-y^{2}}+2 n_{p_{\sigma}}$ in the planes $\left(n_{x^{2}-y^{2}}\right.$ being the concentration of holes of $\mathrm{Cu}-3 \mathrm{~d}$ orbital character and $n_{p_{\sigma}}$ of O-2p orbital character) as well as of a reduced (probably minimum) imaginary susceptibility - as deduced from NMR experiments. In fact, Zheng et al. noticed that the highest $T_{c}$ 's are obtained for a reduced ratio $n_{x^{2}-y^{2}} / 2 n_{p_{\sigma}} \sim 1$, and, moreover, that such a trend is correlated with a reduced relaxation rate $\left(1 / T_{1}\right)_{C u} \propto T \lim _{\omega \rightarrow 0} \sum_{q} A_{q}^{2} \operatorname{Im} \chi(q, \omega) / \omega$, in properly normalized units (here $A_{q}$ is the hyperfine coupling). This last fact points to a reduced $\operatorname{Im} \chi(q, \omega)$ etc. These conclusins are in accordance with our picture, which yields both special values of the filling factor $n$ - for this also c.f. e.g. 8.11 - as well as special small values of the product $\left(a \chi_{A F}\right)$ as a prerequisite for the highest attainable $T_{c}$ 's (also c.f. the last paragraph of section IV).

\section{Summary}

To summarize, we present a single plane Fermi liquid model which for the normal state can explain the salient transport properties, the low energy dependence of $\chi_{M M P}$, and their relation to the existence of van-Hove singularities close to the Fermi surface. E.g. we obtain analytically a scattering rate linear in $\max (T, \epsilon)$, within appropriate $T$ and $\epsilon$ bounds, for all momenta in the Brillouin zone. This result yields directly a linear in $T$ resistivity and linear in $1 / \epsilon$ optical conductivity. The introduction of an ansatz for the susceptibility of the carriers allows for an understanding of both the appearance of the spin resonance peak in the normal state and the temperature behaviour of the Hall conductivity. Further, by using the ansatz we obtain significantly enhanced $d_{x^{2}-y^{2}}$ wave transition temperatures $T_{c}$. Attention is paid to $\mathrm{Nd}_{2-x} \mathrm{Ce}_{x} \mathrm{CuO}_{4+\delta}$, the properties of which, despite appearances, we believe to be fully consistent with those of the majority of cuprates.

In brief, let us discuss the possible connection to the physics of the underdoped cuprates. Strong experimental evidencesuggests that they are in a phase separated regime, with AF domains of spins separated by stripes of holes 3941 . One can envisage that with doping increasing towards the optimal regime, the stripes melt into an effective Fermi liquid, and the physics described here is recovered. Further models on the underdoped cuprates can be found in 60 .

The author has enjoyed discussions with Yia-Chung Chang, Gordon Baym, Joseph Betouras, Girsh Blumberg, Antonio Castro Neto, Lance Cooper, Sasha Liechtenstein, Peter Littlewood, Jörg Schmalian, Raivo Stern, Qimiao Si 
and Branko Stojkovic. This work was supported by the Research Board of the University of Illinois, the Office of Naval Research under N00014-90-J-1267 and NSF under DMR-89-20538.

\section{Appendix - Disorder effects in the susceptibility of the carriers}

Returning to the origin of a small $\omega_{o}$, which was discussed in section IV, another option would in principle be the disorder inherent in the cuprates. The dopants are randomly positioned in the crystal structure, thereby creating an effective disorder potential for the carriers in the planes. We calculated the effect of disorder by considering the dopants as isotropic point scatterers, with a density $n_{i}=1 \%$ and typical scattering strength $V_{s}=0-1 \mathrm{eV}$ (i.e. $\leq 8 t$ ). These parameters give a residual impurity scattering rate less than $2 \mathrm{meV}$, consistent with experiments on the cuprates. For the calculation of the susceptibility we used the diffuson 61 . We only used a band structure with $t^{\prime \prime}=0$, and only took into account non-magnetic disorder. The non-interacting Green's function now becomes

$$
G_{o}^{\prime}\left(k, \epsilon_{n}\right)=\frac{1}{i \epsilon_{n}+\mu-\epsilon_{k}+\sigma_{i}\left(\epsilon_{n}\right)},
$$

with $\sigma_{i}\left(\epsilon_{n}\right)=n_{i} V_{s} /\left(1-\left(V_{s} / N^{2}\right) \sum_{k} G_{o}\left(k, \epsilon_{n}\right)\right)$. The susceptibility is given by

$$
\chi_{o}\left(q, \omega_{m}\right)=-T \sum_{\epsilon_{n}} P\left(q, \omega_{m} ; \epsilon_{n}\right)\left\{\frac{\theta\left(-\epsilon_{n}\left(\epsilon_{n}+\omega_{m}\right)\right)}{1-n_{i} V_{s}^{2} P\left(q, \omega_{m} ; \epsilon_{n}\right)}+\theta\left(\epsilon_{n}\left(\epsilon_{n}+\omega_{m}\right)\right)\right\},
$$

and $P\left(q, \omega_{m} ; \epsilon_{n}\right)=\left(1 / N^{2}\right) \sum_{k} G\left(k+q, \epsilon_{n}+\omega_{m}\right) G\left(k, \epsilon_{n}\right)$. However we found no evidence, for the parameters above, of $\omega_{o}$ being influenced by disorder.

* Current address: 18 Giampoudi St., Iraklio, Crete 71201, Greece. E-mail: kast@iesl.forth.gr .

${ }^{1}$ J. Annett, N.D. Goldenfeld \& A.J. Leggett, in Physical Properties of High Temperature Superconductors, ed. D. Ginsberg, World Scientific, Singapore, vol. V (1996).

${ }^{2}$ D.J. Scalapino, Phys. Repts. 250, 329 (1995).

${ }^{3}$ D. Pines, in High $T_{c}$ Superconductivity and the $C_{60}$ Family, eds. T.D. Lee \& H.C. Ren, Gordon \& Breach (1995).

${ }^{4}$ Y. Iye, in Physical Properties of High Temperature Superconductors, ed. D. Ginsberg, World Scientific, Singapore, vol. III (1992).

${ }^{5}$ A short account of this work has appeared in G. Kastrinakis, Physica C 317-319, 497 (1999); G. Kastrinakis, condmat/9703141.

${ }^{6}$ G. Blumberg, B.P. Stojkovic \& M.V. Klein, Phys. Rev. B 52, R15741 (1995).

${ }^{7}$ O.K. Andersen, A.I. Liechtenstein, O. Jepsen \& F. Paulsen, J. Phys. Chem. Solids 56, 1573 (1995).

${ }^{8}$ N.E. Bickers, D.J. Scalapino \& S.R. White, Phys. Rev. Lett. 62; 961 (1989), N.E. Bickers \& S.R. White, Phys. Rev. B 43, 8044 (1991).

${ }^{9}$ G. Baym, Phys. Rev. 127, 1391 (1962); G. Baym \& L.P. Kadanoff, ibid. 124, 287 (1961).

${ }^{10}$ P. Monthoux \& D. Pines, Phys. Rev. B 47, 6069 (1993), ibid., 49, 4261 (1994).

11 S. Grabowski, M. Langer, J. Schmalian \& K.H. Bennemann, Europhys. Lett. 34, 219 (1996).

${ }^{12}$ Y.M. Vilk \& A.-M.S. Tremblay, Europhys. Lett. 33, 159 (1996).

13 J.P. Carbotte, St. Lenck \& R.C. Dynes, Phys. Rev. B 50, 10149 (1994).

${ }^{14}$ A.I. Liechtenstein, O. Gunnarson, O.K. Andersen \& R.M. Martin, Phys. Rev. B 54, 12505 (1996).

${ }^{15}$ H.J. Vidberg \& J.W. Serene, J. Low Temp. Phys. 29, 177 (1977).

16 A.A. Abrikosov, L.P. Gorkov \& I.E. Dzyaloshinski, Methods of Quantum Field Theory in Statistical Physics, Prentice-Hall (1964).

${ }^{17}$ D. Pines \& Ph. Nozières, The Theory of Quantum Liquids, W.A. Benjamin (1966).

18 J. González, F. Guinea \& M.A.H. Vozmediano, Phys. Rev. Lett. 79, 3514 (1997).

19 The factor $1 / 4$ was kindly pointed out by G. Varelogiannis.

${ }^{20}$ Q. Si \& K. Levin, Phys. Rev. B 44, 4727 (1991). 
${ }^{21}$ D. B. Tanner, private communication.

${ }^{22}$ C.M. Varma, P.B. Littlewood, S. Schmitt-Rink, E. Abrahams \& A.E. Ruckenstein, Phys. Rev. Lett. 63, 1996 (1989); P.B. Littlewood \& C.M. Varma, J. Appl. Phys. 69, 4979 (1991).

${ }^{23}$ H. Kontani, K. Kanki \& K. Ueda, Phys. Rev. B 59, 14723 (1999).

${ }^{24}$ R. Hlubina \& T.M. Rice, Phys. Rev. B 51, 9253 (1995).

${ }^{25}$ B. P. Stojkovic \& D. Pines, Phys. Rev. Lett. 76, 811 (1996)

${ }^{26}$ D.M. Newns, P.C. Pattnaik \& C.C. Tsuei, Phys. Rev. B 43, 3075 (1991).

27 J. González, F. Guinea \& M.A.H. Vozmediano, Europhys. Lett. 34, 711 (1996); Nucl. Phys. B 485, 694 (1997).

${ }^{28}$ R.S. Markiewicz, cond-mat/9611238.

${ }^{29}$ D.H. Lu et al., Phys. Rev. Lett. 76, 4845 (1996).

${ }^{30}$ D.M. King et al., Phys. Rev. Lett. 70, 3159 (1993); R.O. Anderson et al., ibid., 70, 3163 (1993).

${ }^{31}$ C.C. Tsuei, A. Gupta \& G. Koren, Physica C 161, 415 (1989).

${ }^{32}$ Y. Kubo, Y. Shimakawa, T. Manako \& H. Igarashi, Phys. Rev. B 43, 7875 (1991).

${ }^{33}$ D.M. Newns et al., Phys. Rev. Lett. 73, 1695 (1994).

${ }^{34}$ G.C. McIntosh \& A.B. Kaiser, Phys. Rev. B 54, 12569 (1996).

${ }^{35}$ W.H. Beere \& J.F. Annett, cond-mat/9801030.

${ }^{36}$ A.J. Millis, H. Monien \& D. Pines, Phys. Rev. B 42, 167 (1990).

37 S. Sachdev, A.V. Chubukov \& A. Sokol, Phys. Rev. B 51, 14874 (1995).

${ }^{38}$ F. Onufrieva \& J. Rossat-Mignod, Phys. Rev. B 52, 7572 (1995); F. Onufrieva, Physica C 251, 348 (1995).

${ }^{39}$ V.J. Emery, S.A. Kivelson \& O. Zachar, Phys. Rev. B 56, 6120 (1997), and therein.

40 J. Zaanen, cond-mat/9711009, and therein.

41 A.H. Castro Neto, Phys. Rev. Lett. 78, 3931 (1997), and therein.

${ }^{42}$ M. Imada, A. Fujimori \& Y. Tokura, Rev. Mod. Phys. 70, 1039 (1998).

43 A.J. Millis, Phys. Rev. B 45, 13047 (1992).

44 A. Sokol \& D. Pines, Phys. Rev. Lett. 71, 2813 (1993).

${ }^{45}$ H.F. Fong et al., Phys. Rev. Lett. 82, 1939 (1999).

${ }^{46}$ H.F. Fong et al., Nature 398, 588 (1999).

47 N. Bulut, cond-mat/9909437.

48 A.H. Castro Neto \& A. Balatsky, cond-mat/9805273.

${ }^{49}$ D.J. Scalapino, E. Loh,Jr. \& J.E. Hirsch, Phys. Rev. B 34, 6554 (1986).

${ }^{50}$ C. Nayak \& F. Wilczek, cond-mat/9510132.

${ }^{51}$ W. Kohn \& J.M. Luttinger, Phys. Rev. Lett. 15, 524 (1965).

52 J. Betouras \& R. Joynt, Europhys. Lett. 31, 119 (1995).

${ }^{53}$ Note however that our treatment through eq. (29) is inadequate for a quantitative determination of the $s$-wave $T_{c}^{\prime}$, as this occurs deep in the $d$-wave superconducting phase.

54 M. Covington et al., Phys. Rev. Lett. 79, 277 (1997).

${ }^{55}$ K.A. Kouznetsov et al., Phys. Rev. Lett. 79, 3050 (1997).

${ }^{56}$ R. Movshovich et al., cond-mat/9709061.

${ }^{57}$ In terms of FLEX calculations (c.f. section I), the highest $T_{c}$ 's have been obtained by Grabowski, Langer, Schmalian and Bennemann (GLSB) 11 . For $t=250 \mathrm{meV}$ - which we use here as well - they have obtained a maximum $T_{c}=80^{\circ} \mathrm{K}$. They are using a numerical implementation for locating $T_{c}$ (J. Schmalian, private communication) which is different from ours, and which gives systematically higher $T_{c}$ 's than our implementation. E.g. for the same set of optimal parameters of GLSB we obtain $T_{c}=70^{\circ} \mathrm{K}$. All our results from the use of the ansatz (26) should be compared to this latter value.

${ }^{58}$ We note that the basic effect of considering moderate values of an isotropic phonon interaction is to allow for greater values of $U$ before the AF instability condition $b=1$ (c.f. above) sets-in. Hence a finite phonon interaction allows for a bigger on-site/paramagnon coupling.

${ }^{59}$ G.-Q. Zheng, Y. Kitaoka, K. Ishida \& K. Asayama, J. Phys. Soc. Japan 64, 2524 (1995).

60 T. Timusk \& B. Statt, Repts. Prog. Phys. 62, 61 (1999).

${ }^{61}$ P.A. Lee \& T.V. Ramakrishnan, Rev. Mod. Phys. 57, 287 (1985).

\section{FIGURE CAPTIONS}

Figure 1. Full Green's function $G^{R}\left(k_{F}, \epsilon\right)$ for (a) $k_{F}=k_{F}^{\text {boundary }}$ along $(\pi, 0) \rightarrow(\pi, \pi)$ and (b) $k_{F}=k_{F}^{\text {diagonal }}$ along $(0,0) \rightarrow(\pi, \pi)$, for $t=250 \mathrm{meV}, t^{\prime}=-0.11 t, t^{\prime \prime}=0.25 t, U=1.5 \mathrm{eV}, n=0.91$, at $T=105^{\circ} \mathrm{K}$.

Figure 2. Self-energy $\Sigma\left(k_{F}, \epsilon\right)$ for $k_{F}=k_{F}^{\text {boundary }}$ along $(\pi, 0) \rightarrow(\pi, \pi)$ (continuous line) and $k_{F}=k_{F}^{\text {diagonal }}$ along $(0,0) \rightarrow(\pi, \pi)$ (dashed line), for the same parameters as in fig. 1. (a) depicts $\operatorname{Im} \Sigma^{R}\left(k_{F}, \epsilon\right)$ and (b) depicts $\operatorname{Re} \Sigma\left(k_{F}, \epsilon\right)$. A (quasi)linear energy dependence of $\operatorname{Im} \Sigma^{R}\left(k_{F}, \epsilon\right)$ can be seen for energies below $0.5 \mathrm{eV}$ - also c.f. text. 
Figure 3. Self-energy $\Sigma\left(k_{F}, \epsilon\right)$ for $k_{F}=k_{F}^{\text {boundary }}$ along $(\pi, 0) \rightarrow(\pi, \pi)$ (continuous line) and $k_{F}=k_{F}^{\text {diagonal }}$ along $(0,0) \rightarrow(\pi, \pi)$ (dashed line), for $t=250 \mathrm{meV}, t^{\prime}=-0.11 t, t^{\prime \prime}=0.25 t, U=1.47 \mathrm{eV}, n=0.91$, at $T=105^{\circ} \mathrm{K}$. The carrier susceptibility has an antiferromagnetic enhancement according to the ansatz of eq. (26) here (see section IV), with $\xi=1, a X_{o}=0.5 \mathrm{eV}^{-1}$ and $D=32 t$. This is the optimum $T_{c}$ case when using form (B) - eq. (28) - of our ansatz. (a) depicts $\operatorname{Im} \Sigma^{R}\left(k_{F}, \epsilon\right)$ and (b) depicts $\operatorname{Re} \Sigma\left(k_{F}, \epsilon\right)$. A (quasi)linear energy dependence of $\operatorname{Im} \Sigma^{R}\left(k_{F}, \epsilon\right)$ can be seen for energies below $0.5 \mathrm{eV}$.

Figure 4. Evolution of the density of states. Dashed line : non-interacting system, with parameters as in fig. 3. Continuous line : same system with $U=0.8 \mathrm{eV}$. Notice the transfer of the central van-Hove peak towards the chemical potential, the disappearance of the two satellite peaks - see text, and the broadening of the total spectral width. 


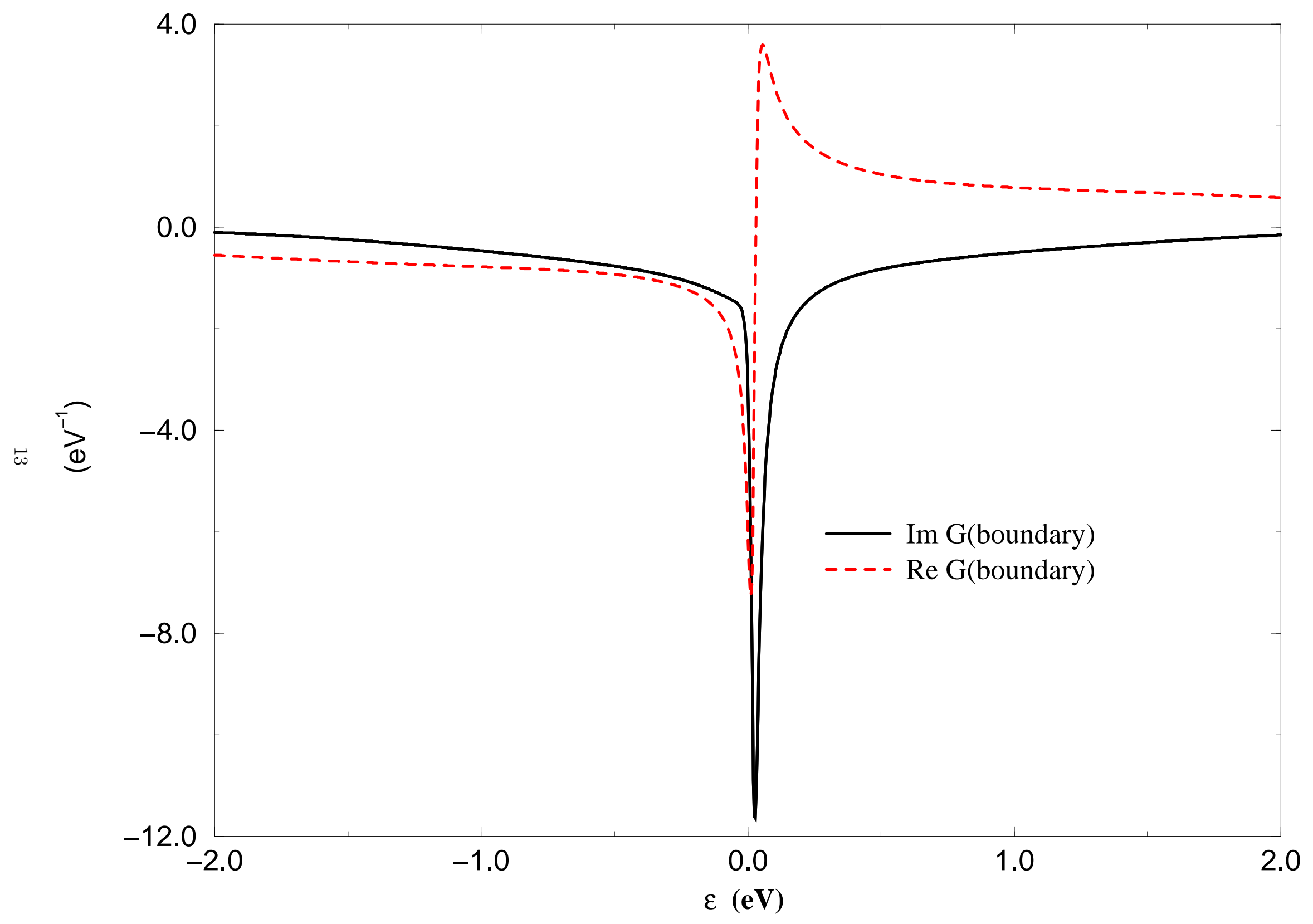




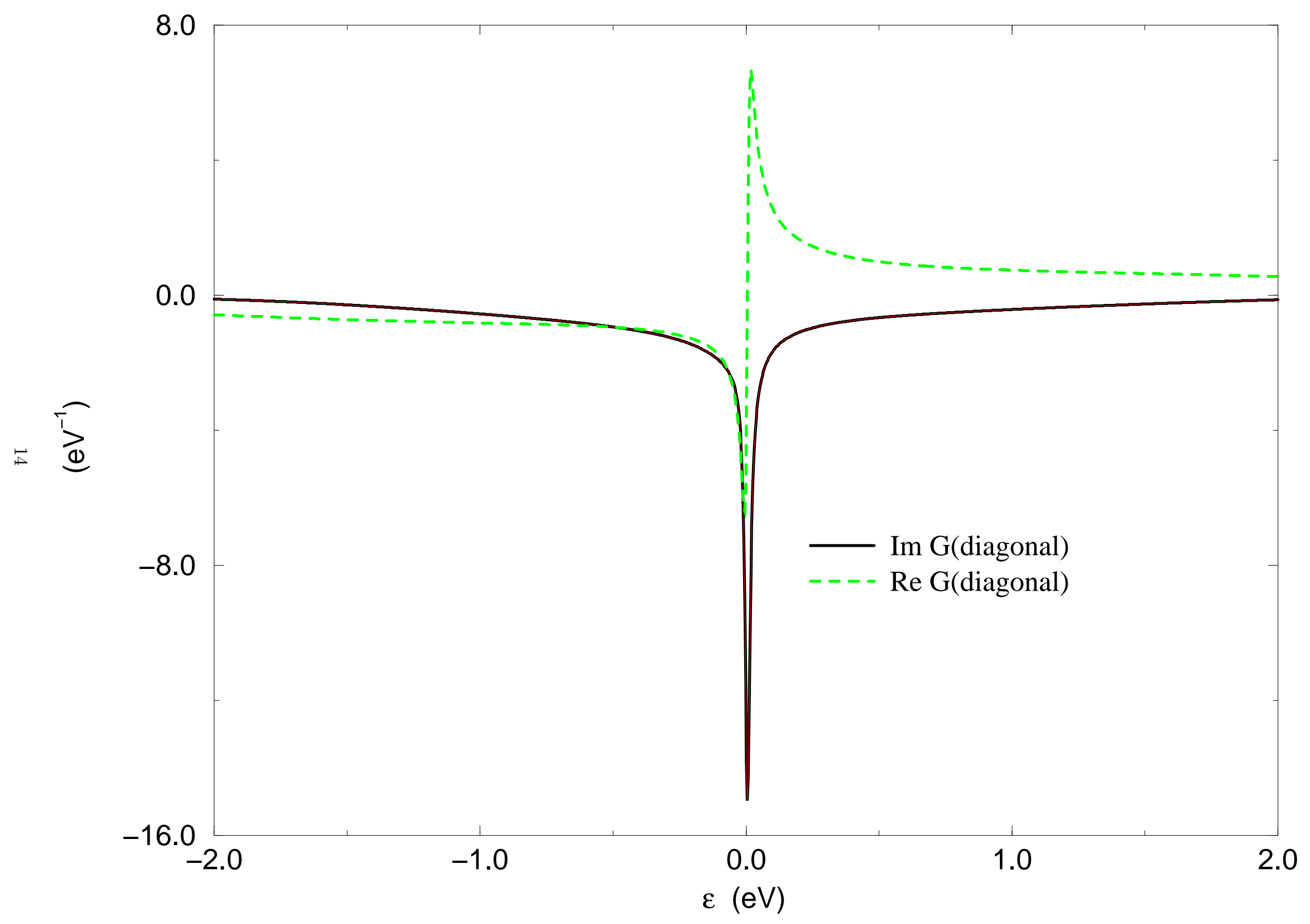




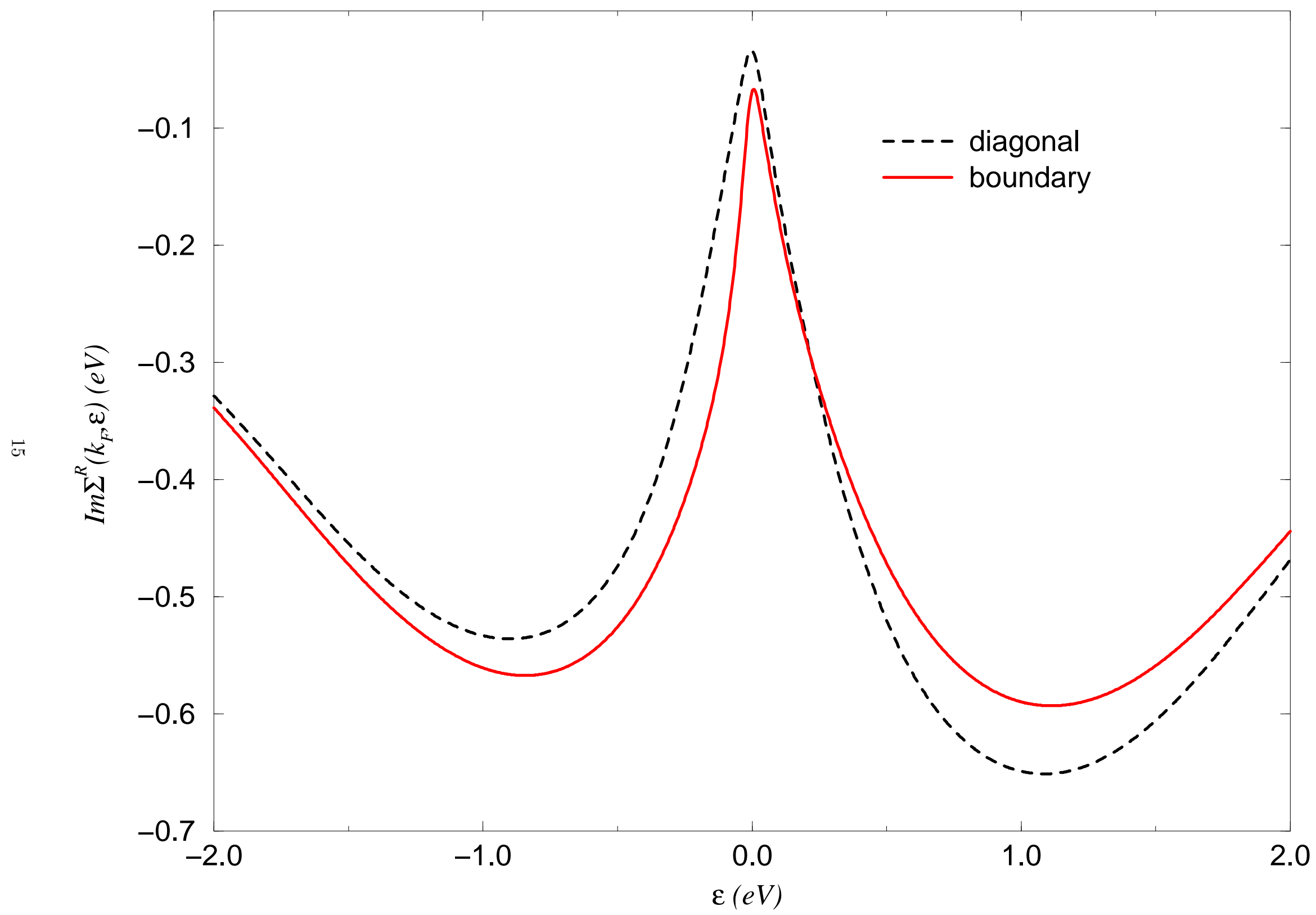




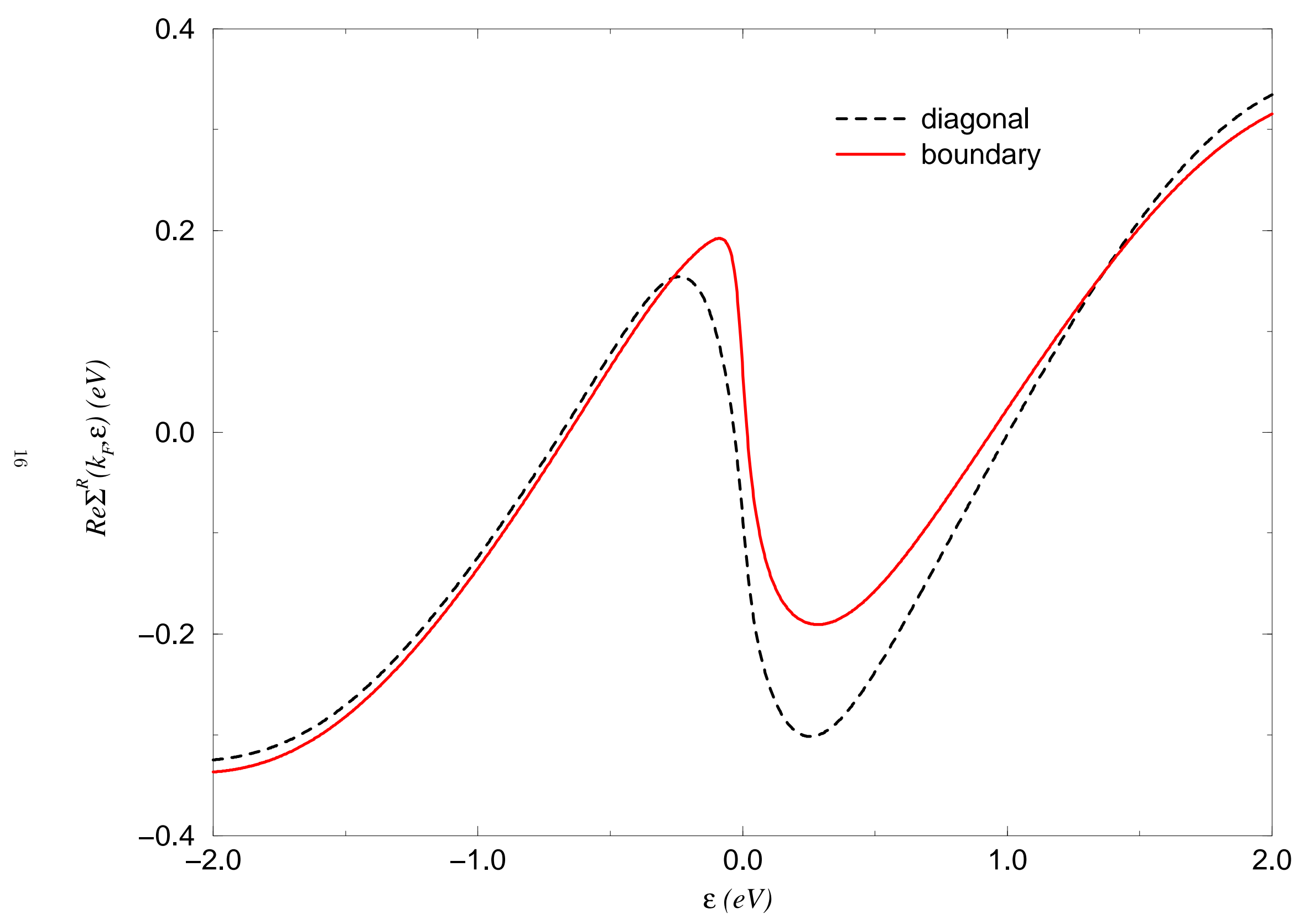




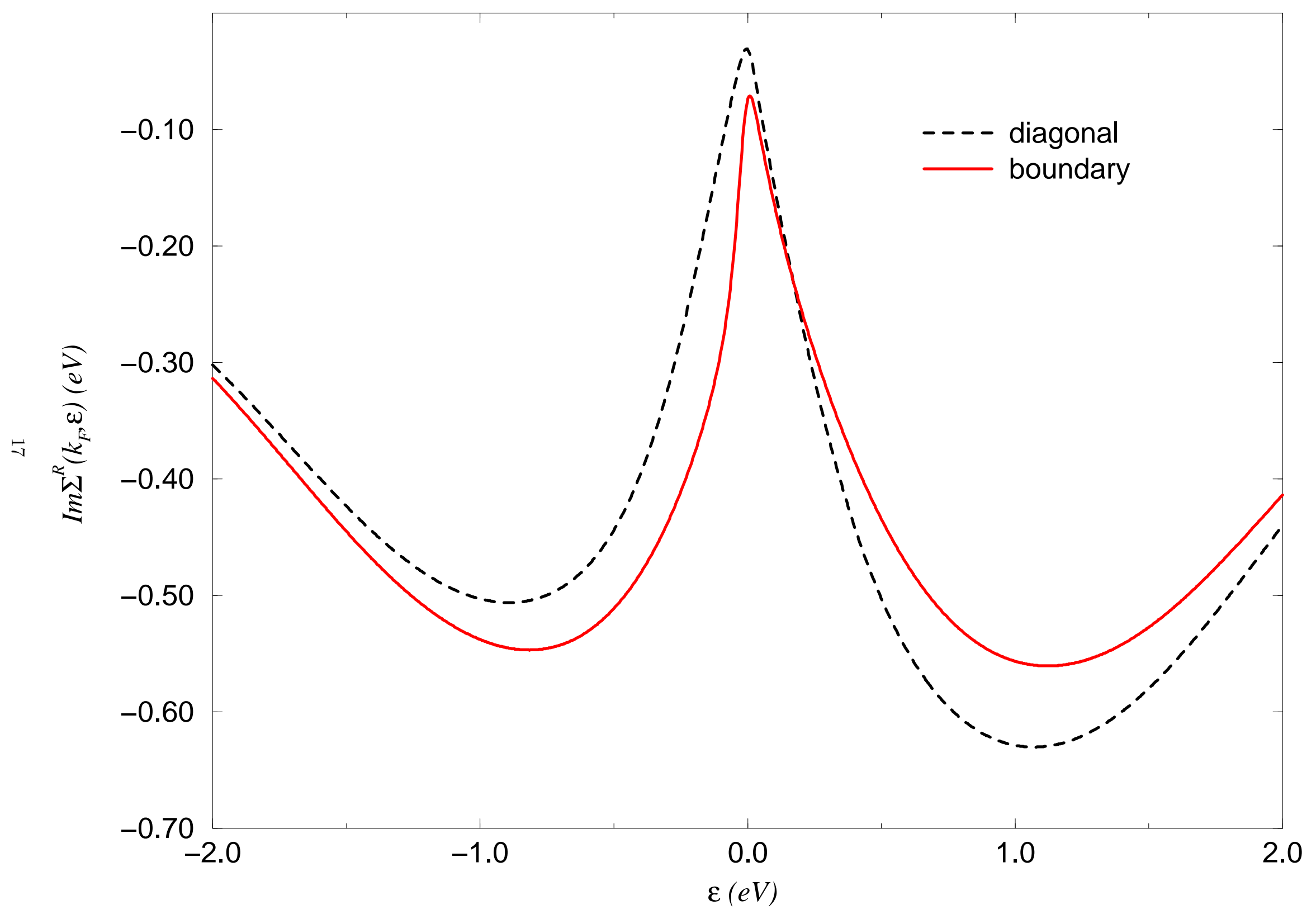




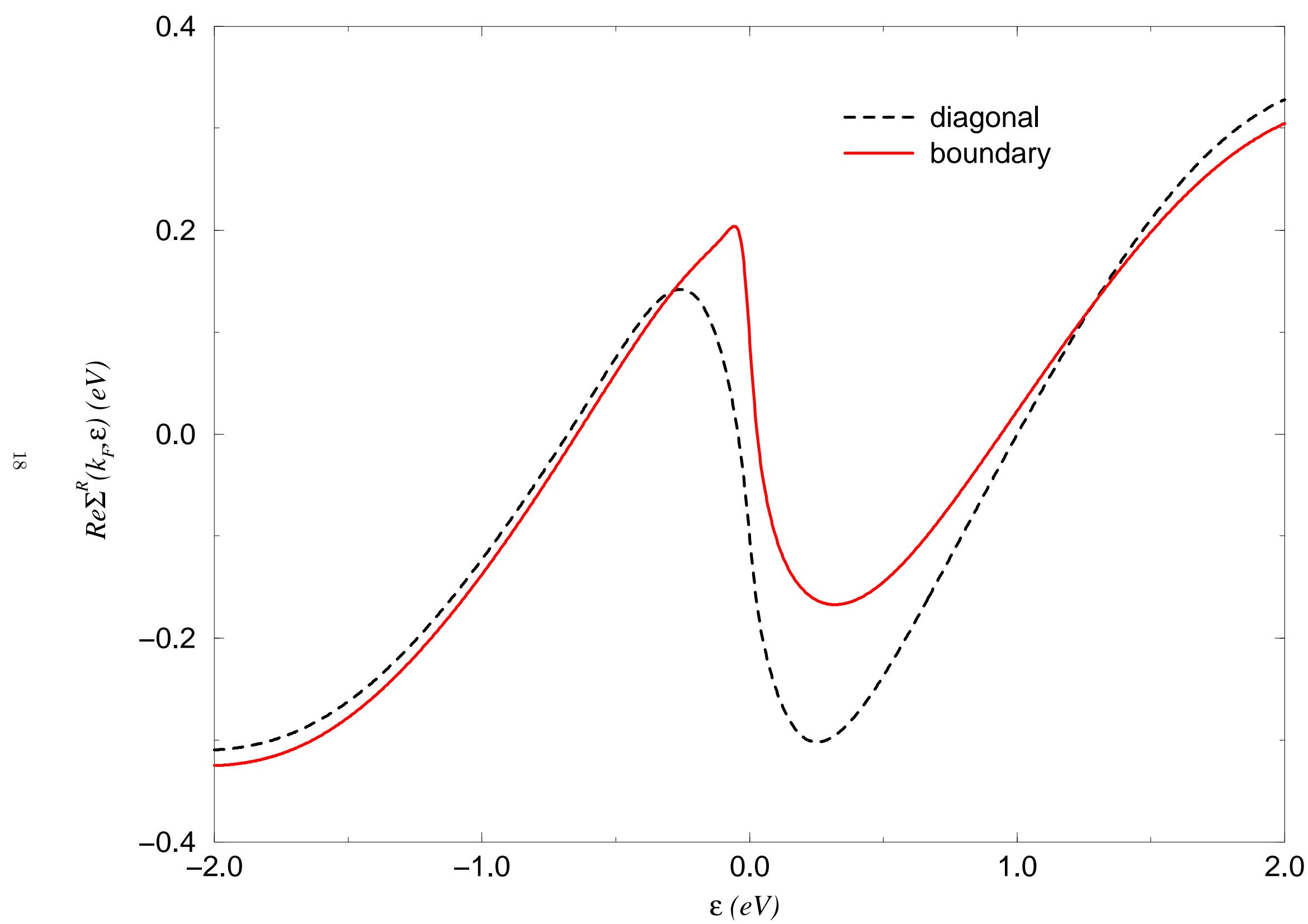




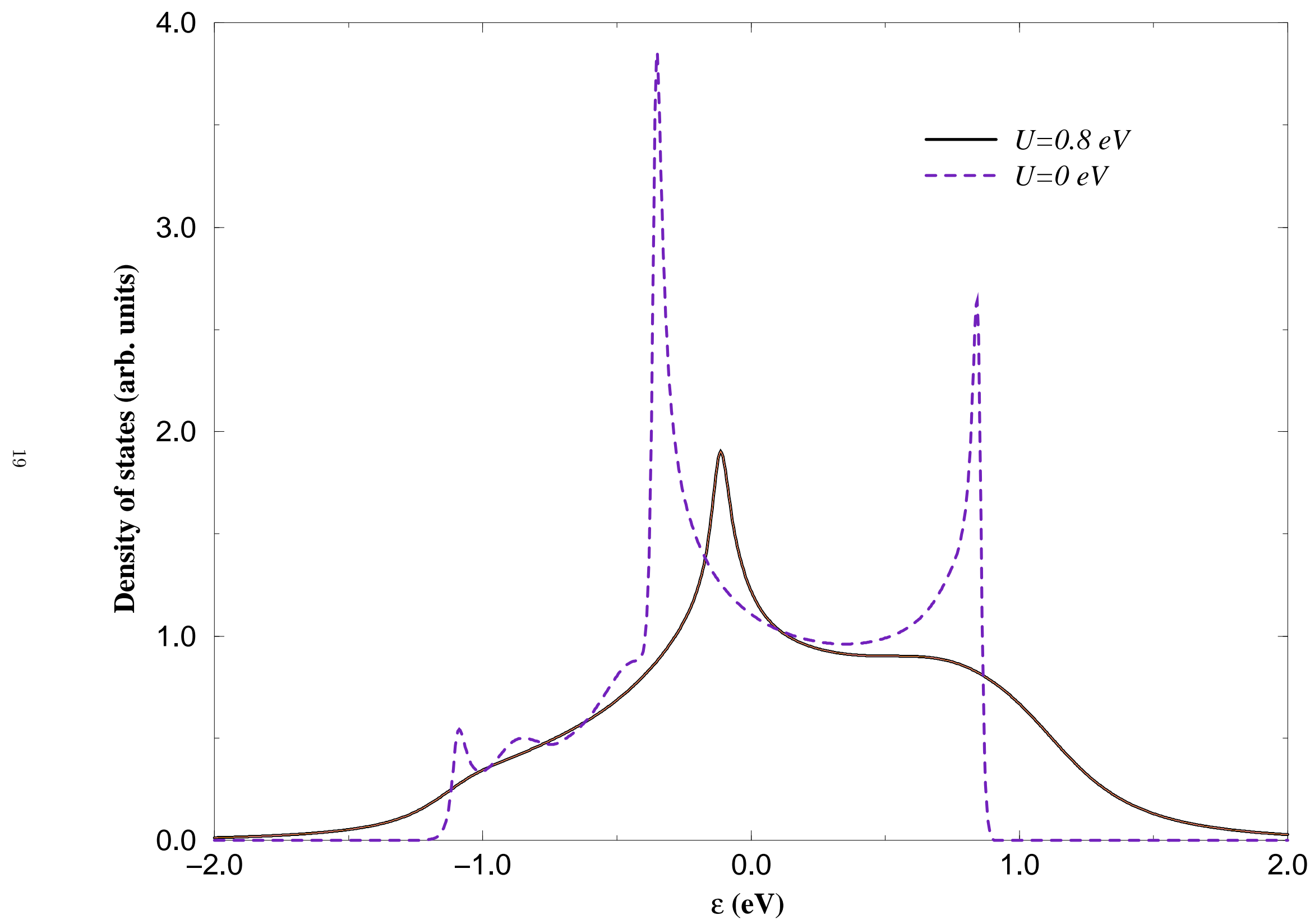

